\title{
Predator Functional Response Changed by Induced Defenses in Prey
}

\author{
Edd Hammill, ${ }^{1,2, *}$ Owen L. Petchey, ${ }^{2}$ and Bradley R. Anholt ${ }^{1}$
}

1. Department of Biology, University of Victoria, P.O. Box 3020, Victoria, British Columbia V8W 3N5, Canada; and Bamfield Marine Sciences Centre, Bamfield, British Columbia V0R 1B0, Canada; 2. Department of Animal and Plant Sciences, University of Sheffield, Western Bank, Sheffield S10 2TN, United Kingdom

Submitted January 6, 2010; Accepted August 3, 2010; Electronically published October 18, 2010

ABSTRACT: Functional responses play a central role in the nature and stability of predator-prey population dynamics. Here we investigate how induced defenses affect predator functional responses. In experimental communities, prey (Paramecium) expressed two previously undocumented inducible defenses - a speed reduction and a width increase-in response to nonlethal exposure to predatory Stenostomum. Nonlethal exposure also changed the shape of the predator's functional response from Type II to Type III, consistent with changes in the density dependence of attack rates. Handling times were also affected by prey defenses, increasing at least sixfold. These changes show that induced changes in prey have a real defensive function. At low prey densities, induction led to lower attack success; at high prey densities, attack rates were actually higher for induced prey. However, induction increased handling times sufficiently that consumption rates of defended prey were lower than those of undefended prey. Modification of attack rate and handling time has important potential consequences for population dynamics; Type III functional responses can increase the stability of population dynamics and persistence because predation on small populations is low, allowing a relict population to survive. Simulations of a predator-prey population dynamic model revealed the stabilizing potential of the Type III response.

Keywords: inducible defenses, predator-prey interactions, functional responses.

\section{Introduction}

Many prey produce defensive phenotypes after exposure to predatory cues (Tollrian and Harvell 1999). These inducible defenses can include changes in behavior, morphology, and life history. Changes in behavioral and morphological traits can occur within or across generations and directly change susceptibility to predation (Van Buskirk and McCollum 2000). Behavioral defensive traits include reduced movement rates (Relyea 2001), leading to

* Corresponding author; e-mail: hammill@zoology.ubc.ca.

Am. Nat. 2010. Vol. 176, pp. 723-731. (C) 2010 by The University of Chicago. 0003-0147/2010/17606-51842\$15.00. All rights reserved.

DOI: $10.1086 / 657040$ reduced encounters between predators and prey and lower consumption rates (Werner and Anholt 1993; Skelly 1994; Van Buskirk and McCollum 2000). Morphological defensive traits include the production of neck teeth in Daphnia pulex (Dodson 1974) and the curved-shell morph in the acorn barnacle (Lively 1986). As well as modifying rates of attack, induced changes in prey morphology can increase the time it takes to handle and digest prey (Jeschke and Tollrian 2000; Altwegg et al. 2006). Therefore, induced changes in prey traits have the potential to alter attack rates and handling times in predator-prey interactions, affecting population dynamics (Vos et al. 2004). Increased handling time lowers maximum predator ingestion rate, lessening the maximum impact of predators on prey and the potential for top-down control and increasing predator-prey dynamic stability. Functional responses describe the number of prey a predator can consume as prey density increases (Solomon 1949; Holling 1959), therefore describing predation risk experienced by prey at different population sizes. In this study, we experimentally examine simultaneous changes in behavior and morphology caused by predator cues and then investigate how the same predator cue affects aspects of the functional response.

When predators handle only one prey at a time, functional responses tend to be either Type II or Type III; Type I functional responses generally are observed in filter feeders (Jeschke et al. 2004). In a Type II response, the proportional rate of prey consumption is highest at low prey densities and decreases as prey density increases. In a Type III response, the proportional rate of consumption is low at low prey densities, initially increases with prey density, then decreases at high prey densities. There is a greater risk of extinction in predator-prey dynamics with Type II than with Type III functional responses, as a Type II functional response results in prey experiencing the highest predation risk at low densities (Sarnelle and Wilson 2008). Evidence exists that differences in time spent collecting prey mediated by optimal foraging decisions or differences 
in habitat/prey type can change the response from a Type II to a Type III (Abrams 1982; Lipcius and Hines 1986).

To investigate how defenses affect functional responses, we used the holotrich ciliate Paramecium aurelia as prey. This species is consumed by the flatworm Stenostomum virginianum (Rhabdocoela, Turbellaria), and the pair coexist in nature (Kratina et al. 2007). Inducible defenses have not been documented in Paramecium, even though this species has been used in predator-prey experiments extensively. A Web of Science literature search including the terms "Parameci* AND (defen* OR induci*)" revealed no papers documenting behavioral or morphological defenses in Paramecium. The search did, however, find papers documenting changes in these traits in other ciliates (Altwegg et al. 2006) and the use of chemical defenses in Paramecium (Sugibayashi and Harumoto 2000), implying that the search was broad enough that papers on morphological or behavioral defenses in Paramecium would have been found if they existed. Inducible defenses are prevalent in other ciliates (Kusch 1993; Hammill et al. 2009) and have been shown to confer benefit in terms of a reduction in predation risk (Altwegg et al. 2006). As Stenostomum readily consumes Paramecium and constitutes a serious predation risk (Kratina et al. 2007), we predicted that Paramecium will induce some form of defense. Hence, the aims of this study were to investigate whether behavioral and morphological changes are found in Paramecium, to measure the effect of induction on the predator's functional response, and to investigate the potential for any observed effects to influence the stability of predator-prey population dynamics.

\section{Methods}

All Paramecium used in this study were the asexually produced descendents of a single individual isolated from a pond on the University of Victoria campus. Paramecium culture media consisted of $0.6 \mathrm{~g} \mathrm{~L}^{-1}$ crushed protozoa pellets (no. 13-2360, Carolina Biological Supply, Burlington, NC) dissolved in Naya mineral water (Mirabel, Quebec) filtered through double-layered no. 4 coffee filters.

Stenostomum were cultured from individuals isolated from a single pond on the University of Victoria campus. They were cultured in Pyrex crystallizing dishes containing $300 \mathrm{~mL}$ Naya water and fed a mixture of bacteria and small flagellates. Individuals of relatively equal length (400-650 $\mu \mathrm{m}$ ) were used in the functional-response trials to minimize differences due to predator size. All experimental subjects were cultured in a controlled-temperature room at $20^{\circ} \mathrm{C}$ on a $16 \mathrm{~L}: 8 \mathrm{D}$ cycle.

The chemical cue used to test for induction of prey defenses consisted of freeze-killed Stenostomum at a density of 250 individuals $\mathrm{mL}^{-1}$. Before being used, this cue was filtered to remove particulate matter; this removed dead predators so that minimal nutrients were added to the experimental vessels as the cue was transferred. Filtering also removes bacteria and small flagellates but does not exclude the possibility that it is these species offered as food that generate the cue. This density of dead predators is higher than naturally encountered densities of live predators because we have observed that, per capita, dead predators generate a weaker response (E. Hammill, personal observation). Stenostomum chemical cue at this density can induce changes in Euplotes that are also preyed upon by Stenostomum (Hammill et al. 2009).

\section{Prey Behavioral and Morphological Defenses}

Control and predator-exposed treatments were distributed across two 24-well plates using a random-number generator. Predator-exposed treatments consisted of $400 \mu \mathrm{L}$ protist media and $200 \mu \mathrm{L}$ predator chemical cue. In control wells, predator cue was replaced with $200 \mu \mathrm{L}$ Naya water. Thirty individual Paramecium were added to each well and left to incubate for $24 \mathrm{~h}$ at $20^{\circ} \mathrm{C}$. All Paramecium used in either the behavioral or the morphological experiments were from the same stock culture and experienced identical environmental conditions before being used. This ensured that differences observed between treatments resulted from experimental rather than initial conditions.

Data on behavioral defenses were collected by videographing each well for 35 s using a Nikon Coolpix connected to a Nikon Optiphot-2 dissecting microscope. Plates were left on the microscope for $20 \mathrm{~min}$ before being videographed, allowing test subjects time to recover after the disturbance associated with transferring the plate to the microscope. Five individuals from each recording were randomly chosen, and their speed was analyzed using ImageJ software (Rasband 2006). Using one video per well ensured that individuals were measured only once. Distance covered was then converted to speed $\left(\mu \mathrm{m} \mathrm{s}^{-1}\right)$. Mean speed per well was used in the analysis, thereby avoiding pseudoreplication. Differences between Paramecium exposed to predator cues and controls were analyzed with a $t$-test.

To measure morphological change, four 24-well plates were established as described above and left to incubate for $24 \mathrm{~h}$. Each well was then fixed with 5\% Lugol's media, and 10 individuals were photographed. This method was employed as dead individuals are easier to photograph, and it ensured that each individual was photographed only once. Lugol's appears to have minimal effects on Paramecium width, and any effect would be similar for both defended and undefended prey. Maximum width was measured using ImageJ, and mean width per well was taken 
as the unit of replication. Differences between control and predator-exposed wells were analyzed with a $t$-test.

\section{Functional Responses}

Foraging models usually assume that the foraging time available to a predator is split between two mutually exclusive activities: searching for prey and handling prey (Holling 1959). Attack rate (area or volume per time) and handling time are what determine the predator's functional response. Type II and III functional responses can be described by the equation

$$
N_{\mathrm{e}}=\frac{P T}{h+\left(1 / b N^{q} N\right)},
$$

where $N_{\mathrm{e}}$ is the number of prey consumed during time $T$ (set to 4 in the current study, to signify the 4 -h duration of the experiment), $N$ is initial prey density, $P$ is the number of predator individuals, and $h$ is handling time (Real 1977), the time taken between stopping searching after an attack to starting searching again. Handling time can include time spent on activities such as capture, subduing, consumption, digestion, and repairs (Jeschke et al. 2002); we therefore treat $h$ as an umbrella parameter encompassing all these activities. Time spent handling prey before rejection or escape also adds to handling time, as it cannot be spent searching for other prey.

The equation above is, effectively, a Type II functional response with a density-dependent attack rate $a=b N^{q}$. Real (1977, p. 294) describes $b N^{q}$ as "the rate of potential encounter (or the detection area swept out) in one unit of time." Parameter $q$ determines the type of functional response, and $b$ is the normalization constant for the scaling of attack rate with prey density. When $q=0$, the equation generates a Type II functional response-attack rate is independent of prey density. When $q>0$, a Type III curve is produced-attack rate increases with prey density.

To produce the defended and undefended individuals for use in feeding trials that can be used to estimate a functional response, we added 30 Paramecium each to the wells of six 24-well plates containing $400 \mu \mathrm{L}$ of protist media. All test subjects were taken from the same stock bottle, ensuring that there were no preexisting differences in behavior, morphology, or reproductive rates. We then randomly added $200 \mu \mathrm{L}$ predator cue to half the wells and $200 \mu \mathrm{m}$ Naya water to the others and incubated for $24 \mathrm{~h}$, mimicking the treatments used in the first experiments. Individuals were transferred from these incubating wells to experimental wells in new 24-well plates. We compared the functional responses of Stenostomum consuming Paramecium that had been exposed to chemical cues (de- fended prey) with those of the control (undefended prey). Five replicates each of $1,3,5,7,10,15,20,30$, or 60 prey were added to $500 \mu \mathrm{L}$ protist media within a well of four 24-well plates (45 samples for predator and control wells; total $n=90$ ). These densities were used, because they more than encompass the range of densities of Paramecium in stock cultures (E. Hammill, personal observation). The large number of trials at low densities makes it possible to distinguish between Type II and Type III, while the very high densities increase the chances of finding the asymptote of the response curve. After prey had been added, one Stenostomum was introduced per well, and the plates were left at $20^{\circ} \mathrm{C}$ for $4 \mathrm{~h}$. As the doubling time for Paramecium at $20^{\circ} \mathrm{C}$ is $\sim 12 \mathrm{~h}$ (Leary and Petchey 2009), reproduction during the experiment would be small. Each well was then fixed with three drops of 5\% Lugol's media, and unconsumed Paramecium were counted. It is possible that during the time course of the predation trial, the "undefended controls" may have started to initiate defenses. However, as the density of predators in the trial was relatively low (1 per $500 \mu \mathrm{L}$ ) this effect should be small. Nonetheless, when we use the term "undefended prey," we are referring to the prey that had not been preexposed to predator cues.

We wished to examine how exposure to predator cues affected the functional response parameters $b, h$, and $q$. In our experiment, and many others, prey density $N$ was not constant but rather declined throughout the experiment due to predation. Therefore, to find the predicted number of prey eaten, we used numerical integration of declining prey density over the same duration as the experiment to find the number (and therefore the proportion) of prey consumed. Using R (R Developmental Core Team 2009), we fitted by maximum likelihood with binomial errors eight versions of this model to the data from both treatments. In the full model, six parameters were estimated: each of the three parameters $b, h$, and $q$ was estimated for each of the two treatments (defended and undefended). We then fitted three models in which one of $b, h$, and $q$ was constant across the two treatments and the other two parameters varied between treatments (i.e., five parameters were estimated, one of which was the same for defended and undefended treatments). We then fitted three models where only one parameter varied between treatments (e.g., two estimates of $b$, one for each treatment, and only one estimate of $h$ and of $q$; a four-parameter model). In the final model, one estimate was made for all three parameters, resulting in a single functional-response model being fitted to data from both treatments (a threeparameter model). Modeling with more parameters generally improves fit with the cost of increased complexity. Models were therefore compared using the Akaike Information Criterion (AIC) and AIC weights (AICw; Wagenmakers and Farrell 2004) to determine each model's 
relative support. The AIC strikes a balance between fit and parameter number (Burnham and Anderson 2002).

We also performed two likelihood ratio tests, in order to illustrate the robustness of the conclusions on the basis of AIC. First, we compared the likelihood of the sixparameter model to that of the five-parameter model in which $q$ was not estimated separately for each treatment. This tests whether a single shape of functional response can explain the observed data from both treatments or whether different functional response types are required for the two treatments. We also used a likelihood ratio test on data from the defended treatment only. This tested whether, for only this half of the data set, a Type II or III functional response was a significantly better explanation of the observed data.

\section{Consequences for Population Dynamics}

Any change caused by prey defenses observed in the functional response may or may not contribute to population stability. To illustrate any stabilizing effect of defenses and changes in the functional response, we simulated population dynamics using a straightforward Lotka-Volterratype model (Lotka 1925):

$$
\begin{aligned}
& \frac{d N}{d t}=r N\left(1-\frac{N}{K}\right)-P N f, \\
& \frac{d P}{d t}=\partial P R f-P m .
\end{aligned}
$$

Equation (2) describes the dynamics of prey density $(N)$ and equation (3) the dynamics of predator density $(P)$. Paramecium species display logistic growth in the absence of a predators; logistic growth is therefore the first term describing prey population dynamics (intrinsic growth rate $r$ and carrying capacity $K)$. The second term is loss to predation described by the functional response $f(N)=$ equation (1) without the $P$ and $T$ terms. Predator dynamics are described by gains from predation, with $\partial$ as the conversion efficiency, and loss to mortality, with $m$ as predator death rate. We simulated population dynamics by numerical integration for 500 time steps ( $\sim 500$ prey generations) and then calculated, for the last 10 time steps for both the prey and predator populations, a measure of population stability: the inverse of the coefficient of variation (Lehman and Tilman 2000).

There were three contrasting simulations with different values of attack rate, handling time, and $q$ : (1) with the Type II functional response estimated from the undefended prey (and $q=0$ ), (2) with the Type II functional response estimated from the defended prey (and $q=0$ ), and (3) with the Type III functional responses estimated from the defended prey $(q=1.52)$. Other parameters were taken from distributions with ranges corresponding to estimates from previous studies $(r=$ uniform $[0.4,1]$, $K=$ uniform $[10,20], \partial=$ uniform $[0.1,1], m=$ uniform [0.08-0.12]). Each of the three contrasting simulations was carried out 100 times, in order to assess the importance of the different functional responses relative to the uncertainty in other parameter values. To illustrate differences in the population dynamics themselves, we took a single estimate of parameters from the random, plausible distributions, so $r=0.7, K=18.01, \partial=0.43$, and $m=0.09$, and plotted the dynamics of equations (2) and (3) with functional responses derived from our data.

\section{Results}

\section{Prey Defenses}

Exposure to chemical cues from Stenostomum reduced Paramecium speed by $53.14 \% \pm 3.05 \%$ (fig. $1 a ; t_{21}=$ $7.94, p<.001)$ and increased body width by $38.00 \% \pm$ $7.67 \%$ (fig. $1 b ; t_{21}=8.36, p<.001$ ).

\section{Predator Functional Responses}

Exposure to predator chemical cues caused marked changes in the predator's functional response. The full model with separate estimates of parameters $b, h$, and $q$ for undefended and defended Paramecium had the lowest AIC (by >3 AIC units; table 1; fig. 2) and an AIC weight of $76.5 \%$ (table 1), a level of support 5.5 times that of the next best model. In the control treatment, parameter $q$ was not significantly different from 0 , indicating a Type II functional response (table 2). However, in the case of defended prey exposed to predator cues, parameter $q$ was more than 2 SE greater than 0 (table 2), indicating a Type III functional response. A likelihood ratio test indicated that the full model was significantly better than the model in which the shape of the functional response was not allowed to depend on treatment level $\left(\chi_{(1)}^{2}=5.52, p=\right.$ .023). Focusing on defended prey only, a likelihood ratio test showed a significant difference between the fit of models where $q$ was set to 0 and those in which $q$ was a free parameter $\left(\chi_{(1)}^{2}=5.49, p=.019\right)$, providing further evidence that $q$ was different from 0 and that Stenostomum feeding on induced Paramecium had a Type III functional response.

The consequences for attack rates attributed to the difference in $q$ between the undefended and the defended prey is illustrated in figure 3 . For undefended prey with a Type II response $(q \approx 0)$, attack rate is independent of prey density; in contrast, defended prey, with $q=1.52$, 
a)

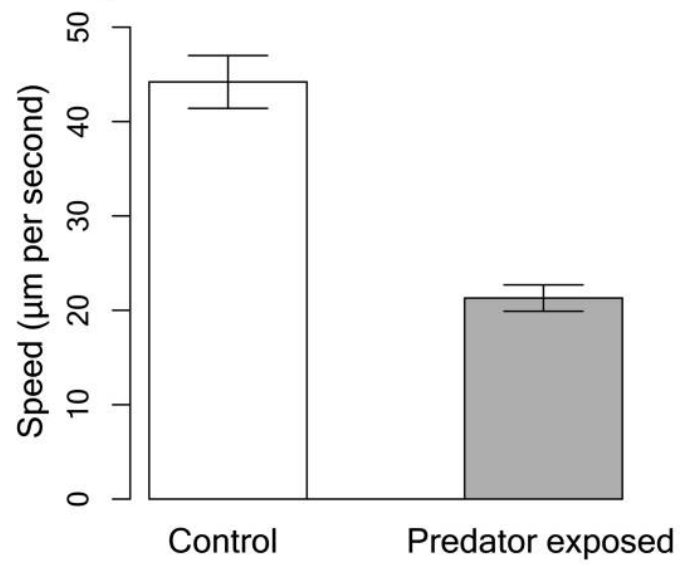

b)

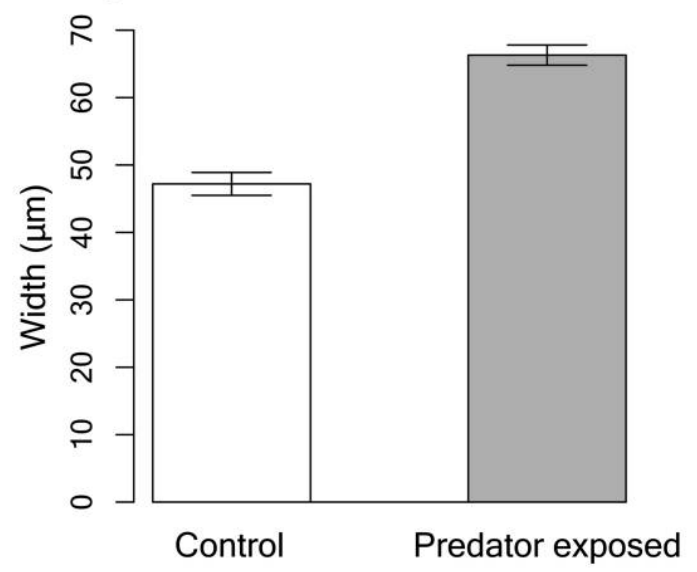

Figure 1: Changes in two traits of Paramecium after exposure to cues from predators: $a$, differences in speed while foraging between the two treatments; $b$, changes in Paramecium body width in the two treatments. Error bars are \pm 1 SE.

experience a greatly reduced attack rate at low prey density and a greatly increased attack rate at high prey densities (fig. 3). If a Type II response is fitted to the defended-prey data, attack rates are represented by parameter $b$ and are constant across prey densities at a value of $b=0.05$.

When the full model is fitted to the data, defended prey have a lower value of parameter $b$, and this, combined with $q=1.52$, means that they experience low consumption rates at low densities (fig. 3). Prey showing inducible defenses also have a greater value of parameter $h$ (handling time), representing an $\sim 6.5$-fold increase in handling times (table 2).

Table 1: Ability of different models to explain the observed data

\begin{tabular}{lcccc}
\hline $\begin{array}{l}\text { Model parameters } \\
\text { estimated separately } \\
\text { for each treatment }\end{array}$ & AIC & $\begin{array}{c}\text { Number of } \\
\text { parameters }\end{array}$ & $\Delta$ AIC & $\begin{array}{c}\text { Weight } \\
(\%)\end{array}$ \\
\hline$b, h, q$ & 230.1 & 6 & .0 & 76.50 \\
$b, h$ & 233.5 & 5 & 3.4 & 13.90 \\
$h, q$ & 237.6 & 5 & 7.5 & 1.80 \\
$b, q$ & 237.0 & 5 & 6.9 & .70 \\
$h$ & 236.3 & 4 & 6.2 & 3.50 \\
$b$ & 276.5 & 4 & 7.5 & $<.01$ \\
$q$ & 237.5 & 4 & 7.4 & 1.90 \\
None & 294.0 & 3 & 63.9 & $<.01$ \\
\hline
\end{tabular}

Note: Models that did not estimate a parameter separately for each of the two treatments (e.g., parameter $q$ for the model in the second row) were forced to estimate only one value of this parameter across the entire data set (i.e., both treatments). The Akaike Information Criterion (AIC) is the likelihood of a model discounted by its complexity (number of parameters); $\triangle \mathrm{AIC}$ is the difference between the AIC of a model and the AIC of the lowest-AIC model; weight is the percentage support for a particular model calculated according to the difference in AIC between the lowest-AIC model and each of the others.

\section{Consequences for Population Dynamics}

Simulated dynamics indicated that the population stability of prey and predator was greater when the model included the Type II-defended than when it included the Type IIundefended functional response (fig. $4 a, 4 b$ ). Population stability was further increased when the modeled functional response corresponded to the Type III response estimated from the defended data set (fig. $4 a, 4 b$ ). The increase in stability resulting from the Type III functional response was large relative to the variation in population stability caused by uncertainties in other parameter values (fig. $4 a, 4 b$ ). Plotting simulated data also illustrates both the qualitative differences in population dynamics when defended prey have a Type III or a Type II functional response and the comparison with undefended prey (fig. $4 c-4 e$ ). Regardless of defense level, fitting a Type II response produced damped oscillations (fig. $4 c, 4 d$ ); however, they settled to equilibrium faster for defended prey. When the Type III response was fitted to defended prey, no oscillations were observed (fig. 4e). Prey defenses also appear to result in higher predator and prey equilibrium densities (fig. $4 c-4 e$ ).

\section{Discussion}

The behavior and morphology of Paramecium and the functional response of the consumer Stenostomum were fundamentally altered by preexposure of prey to predator cues. Changes in the functional response were consistent with the decreased swimming speed and increased size of Paramecium preexposed to predator cue. We believe that this represents the first demonstration of morphological 


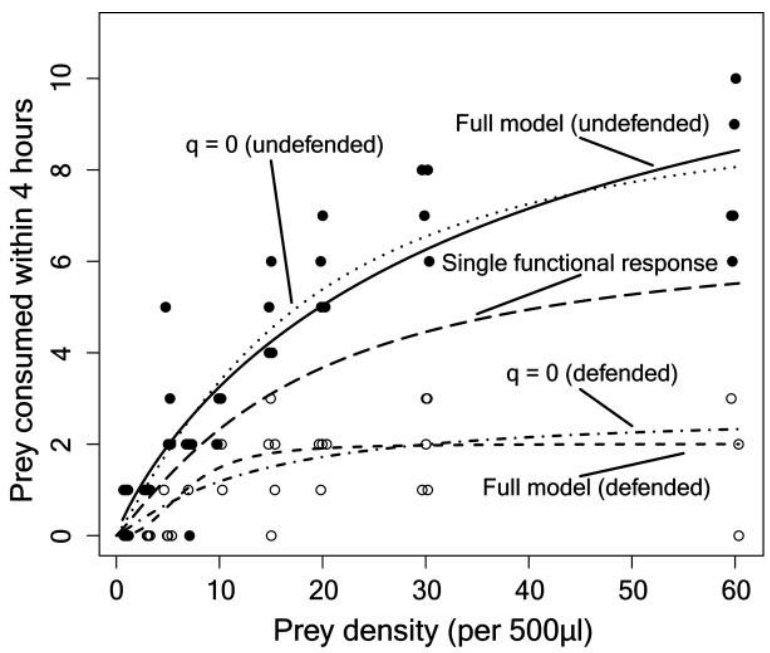

Figure 2: Functional responses generated by fitting the Real (1977) predator equation to the data and allowing different sets of parameters to vary between defended and undefended prey. For clarity, only the full model and two others are displayed. The $q=0$ lines represent the model where a single value of parameter $q$ was used for both defended and undefended prey. Maximum likelihood estimation produced a value not significantly different from 0 , fitting a Type II curve to both treatments. Raw data points are graphed; filled circles represent undefended prey, and open circles represent defended prey. Data points have some random horizontal displacement added for clarity.

and behavioral defenses in Paramecium, as well as the benefits of these defenses. In addition, this appears to be the first demonstration of induced defenses changing the form of a functional response, from Type II to III. Inducible defenses without time lags theoretically stabilize predator-prey interactions due to increased handling times (Vos et al. 2004), and the change from a Type II to a Type III may be another route by which inducible defenses stabilize population dynamics.

The functional response is defined by three parameters, $b$ (attack rate), $h$ (handling time), and $q$; the last dictates whether the functional response will be Type II $(q=0)$ or Type III $(q>0)$. All three of the $b, h$, and $q$ parameters were different for defended and undefended prey (table 2 ), indicating that defense induction substantially changes the predator's functional response. The change in the shape of the functional response, from Type II to Type III, was indicated by the change in parameter $q$. In a Type III response, attack rates are related to parameters $b$ and $q$ and are prey-density dependent (fig. 3). In a Type II response, attack rates are related only to parameter $b$ and are constant across prey densities. For Stenostomum feeding on undefended Paramecium, $q \approx 0$, indicating a Type II functional response. In contrast, for defended Paramecium, $q>0$, producing a Type III functional response.
This change from a Type II to a Type III is due to low attack rates at low prey densities (fig. 3), meaning that relative predation risk is low. The low relative predation risk at low densities (fig. 3) means that a lower prey birthrate is required to result in positive prey population growth at low prey density, making for broader conditions that can result in prey population persistence. In a Type II response, the greatest relative risk of predation is at low densities, meaning that small populations suffer a relatively large risk of extinction (Sinclair et al. 1998; Sarnelle and Wilson 2008).

While, in theory, Type III functional responses are stabilizing relative to Type II, correlated changes in other parameters or relatively little time spent at low prey population density may reduce or even negate this stabilizing effect. The simulations that we performed indicate a strong stabilizing effect of the Type III functional response per se. Changes in handling time associated with the induced defense caused an increase in population stability, but the change to a Type III caused a large additional stabilizing effect, both quantitatively in terms of increasing the inverse of the coefficient of variation and qualitatively in terms of removing the cycling-damped oscillations. Oscillations increase the potential for populations to become extinct, because at low densities, stochasticity may mean that the population level drops below 0 (Gurney and Nisbet 1998). This probability of extinction will be greater in the undefended prey through the combined effects of the greater magnitude of oscillation and the increased number of cycles. This indicates that induced defenses can stabilize predator-prey dynamics via two mechanisms: increased handling times and a shift to a Type III functional response.

There are several potential explanations for the switch from a Type II to a Type III response, and although testing among mechanisms was not the goal of our research, some guarded speculation is possible. The density dependence of attack rates by Stenostomum on defended prey could

Table 2: Parameter estimates for the full model fitted to the data

\begin{tabular}{lcl}
\hline Parameter & Estimate & SE \\
\hline$b_{\text {undefended }}$ & .17 & .065 \\
$b_{\text {defended }}$ & .0047 & .0049 \\
$h_{\text {undefended }}$ & .29 & .16 \\
$h_{\text {defended }}$ & 1.96 & .37 \\
$q_{\text {undefended }}$ & -.13 & .25 \\
$q_{\text {defended }}$ & 1.52 & .72 \\
\hline
\end{tabular}

Note: Forty-five microcosms were used for defended and undefended prey, giving a total sample size of 90 . Handling time (h) is in hours and appears realistic considering the number of prey consumed at high prey densities; $b$ and $q$ contribute to attack rate; actual attack rates are shown in figure 3. 


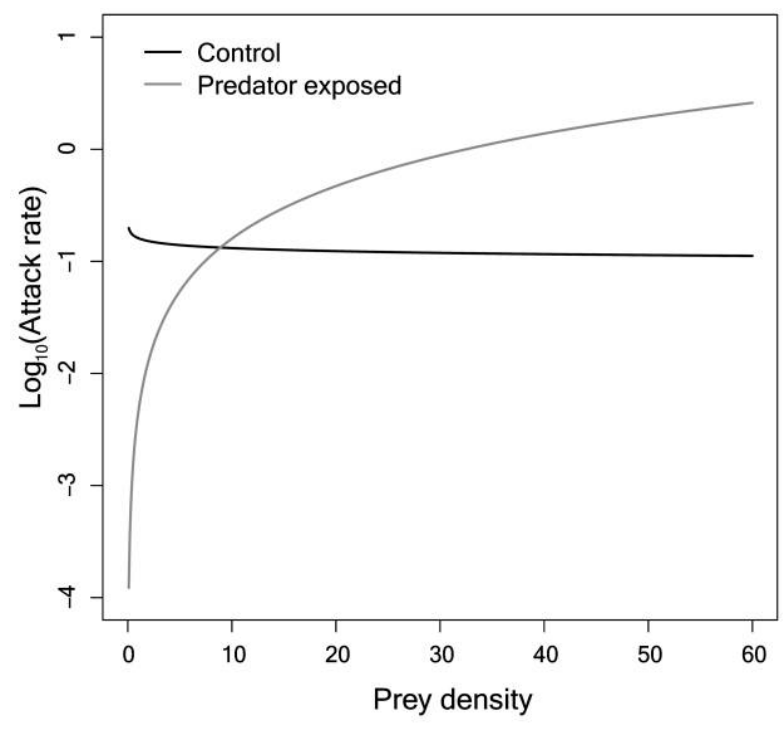

Figure 3: Modeled attack rates $\left(b N^{q}\right)$ estimated from the functionalresponse data, for control (undefended) and predator-exposed (defended) prey. The most parsimonious model of the data is of densitydependent attack rates for predator-exposed prey $(q=1.52)$ and constant attack rates for undefended prey $(q=0)$. Attack rate is defined as the rate of potential encounter; units are volume per unit of time.

happen if prey adjust their behavior depending on the number of prey present. If prey densities in the experimental wells are low, meaning that risk of consumption is relatively high, Paramecium could reduce their speed to a greater extent than at high prey densities. This densitydependent speed reduction could be responsible for the lower attack rate at low prey densities (fig. 3). This explanation for the change in functional-response type highlights the need for further research into density dependence of prey defensive traits (Schaffner and Anholt 1998; Wiackowski and Staronska 1999).

The asymptote of the functional response was also greatly changed by exposure to predator cue. Handling time determines the height of the asymptote of the functional response, that is, the maximum prey-ingestion rate. This change in handling time is likely related to the width increase observed in predator-exposed Paramecium. Morphological defenses have been shown to increase handling times due to the increased time required to capture, subdue, and digest prey (Jeschke and Tollrian 2000; Altwegg et al. 2006). Time spent handling during failed attacks increases handling time, as it is time that cannot be spent searching for new prey. As there is a potential association between increased handling time and chance of escape, it may be selected for at the individual level.

Changes in predator behavior may also explain the change in the functional response. Type III functional re- sponses were initially associated with vertebrate predators where learning played an important role, until several examples were documented in invertebrates (Hassell et al. 1977). In particular, learning was the prey-density-dependent formation of search images. Learning capacity of an organism such as Stenostomum may be limited, although we cannot rule out that it could have been responsible for higher attack rates at high prey densities, particularly in the induced treatment, when prey were larger.

It is also conceivable that prey-density-dependent changes in handling time were in part responsible for the change to a Type III response: defended individuals are likely harder to handle, and Stenostomum may require some acclimatory or learning time to handle them faster. Preydensity-dependent handling times are not usually considered in studies of functional responses. However, partial consumption can be more common at high prey densities, and feeding may become "sloppier." This should lead to a reduction in handling times at high prey densities (Sih 1980), and further studies should address this possibility directly. Therefore, it is possible that density dependence of handling time could contribute to the observed shift from a Type II to a Type III response, and separating the effect of this from the effect of density-dependent attack rates should be a goal of subsequent experiments.

In a previous functional-response study, Altwegg et al. (2006) showed that Stenostomum consuming Euplotes, regardless of defense level, had a Type III functional response. The differences in the functional responses between Stenostomum feeding on Paramecium and those feeding on Euplotes may be related to differences in morphology. Undefended Euplotes are still relatively wide and therefore harder to consume (Altwegg et al. 2006). As they are harder to consume, there may again be some form of learning time associated with them producing a Type III response regardless of defense level. In addition, if predators abandon a predation attempt on defended prey sooner, to allow searching for another prey item when prey are abundant, this will increase the apparent attack rate (Anholt et al. 1987). This increase could lead to the pattern of attack rates observed here, where attack rates for defended prey were higher than those for undefended at high densities (fig. 3). However, as handling times were so much higher $(\sim 6.5 \times)$ for defended prey, although they were attacked more often, the increased handling times mean that actual consumption rates were lower.

There is also the potential that after cue exposure, some prey may become so large as to be invulnerable. Across the duration of the experiment, consumption of susceptible prey would result in a proportional increase in invulnerable prey. However, this alone would be insufficient to produce a Type III response, as the numerical-integration method of analysis accounts for prey depletion. At 


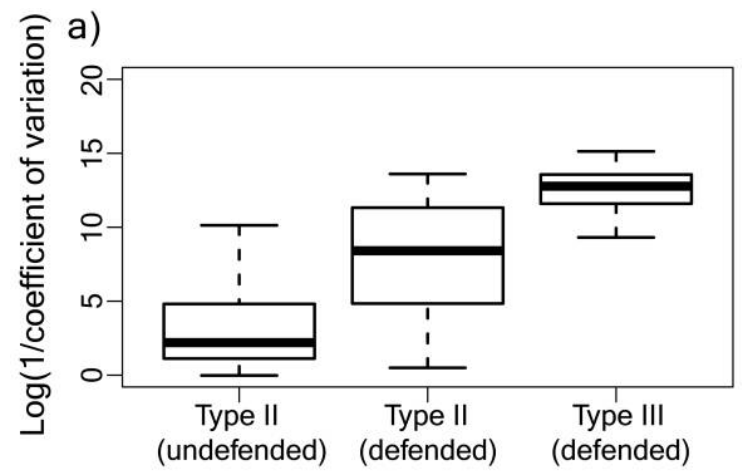$$
\text { ) }
$$

c)

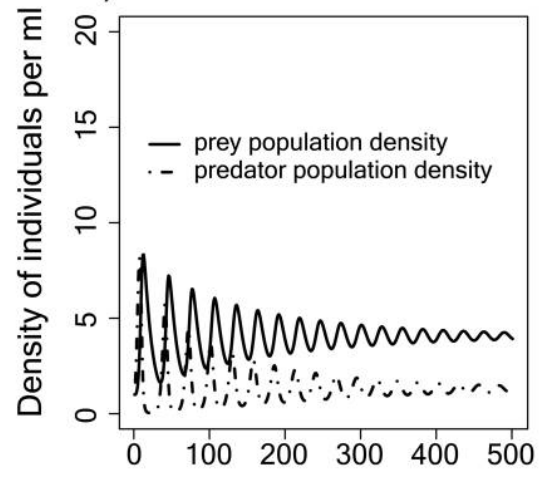

b)

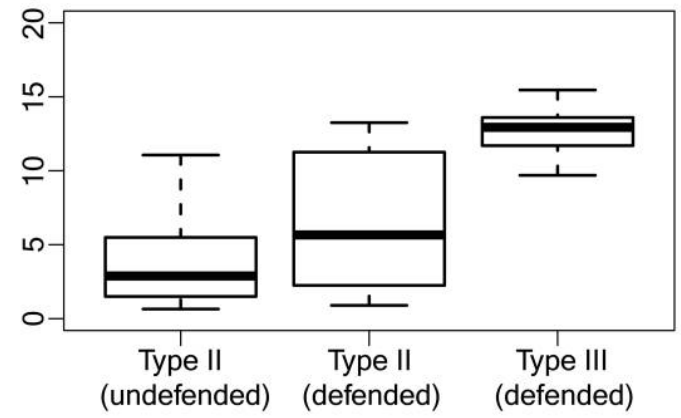

e)
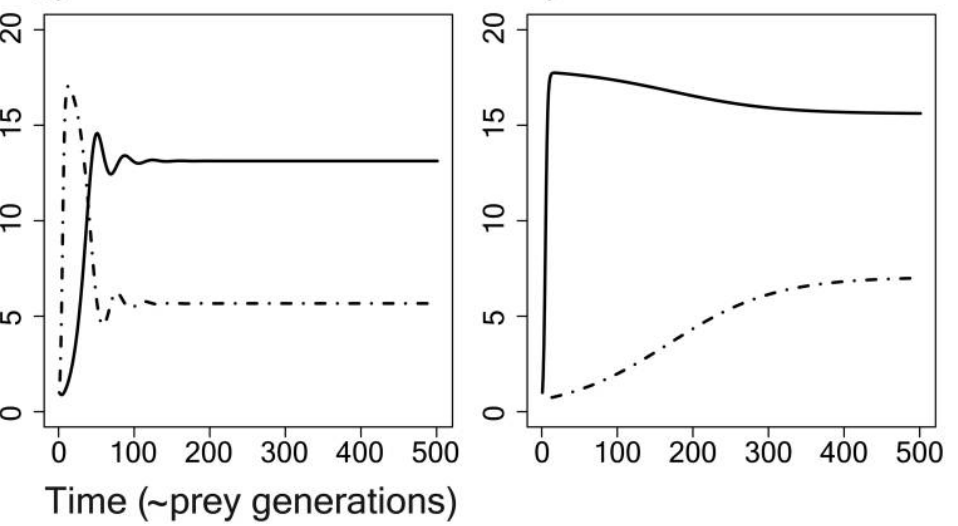

Figure 4: $a, b$, Population stability generated from simulations of predator-prey population dynamics with different defense treatments and estimated functional response type: prey population stability $(a)$; predator population stability $(b)$. $c-e$, Illustrative dynamics of predator and prey population densities generated from a Lotka-Volterra-type model incorporating functional responses derived from our empirical data: undefended prey $(c$, Type II); defended prey ( $d$, Type II); defended prey ( $e$, Type III). See the text for further information.

present, these potential mechanisms are speculative, requiring validation, and may be a productive area for future study.

Previous work on other ciliate species being preyed on by Stenostomum shows a complex temporal interplay between the expressions of behavioral and morphological defenses (Hammill et al. 2010). In our study, this time course of defenses was not measured, but control individuals in the functional-response experiment may have induced some behavioral changes and started production of morphological defenses. However, due to the short duration of the experiment, morphological changes ought to be minimal, and we observed no obvious differences here (E. Hammill, personal observation). Any induction of undefended prey during the functional-response trial would likely reduce the effect of preexposure to predators, making our results, if anything, conservative.

In addition, we did not examine how the magnitude of defense expression is related to predator or prey density, which, as we stated above, may be driving the switch from a Type II to a Type III. Densities of both predators and prey have been shown to affect defense levels in other ciliates (Wiackowski and Staronska 1999) and may affect the form of the functional response we observed. This is the first time that inducible behavioral and morphological defenses have been documented in Paramecium, and investigations into density dependence may be a good direction for future study.

In summary, we show inducible defenses for the first time in Paramecium and illustrate how preexposing prey to the threat of predation can alter the predator's functional response. By adopting a rigorous analytical approach, it was possible to relate different defensive traits to the aspects of predation they affect. We were then able to speculate about the relationship between a defensive trait and the potential form of protection it confers. In the future, it may be possible to make the model fully mechanistic by clarifying the link between its parameters, the observed behavioral and morphological defenses, and possible dependence of defense induction on prey density. This would allow the mechanistic relationships between defense and functional response to be better explored and 
a deeper understanding of predator-prey dynamics to be provided.

\section{Acknowledgments}

This work was funded by the Canada Research Chairs program and a Natural Sciences and Engineering Research Council Discovery Grant awarded to B.R.A. The authors would like to thank B. Bolker for his invaluable help with aspects of the analysis and P. Abrams for comments on an earlier version of the manuscript. O.L.P. is a Royal Society University Research Fellow.

\section{Literature Cited}

Abrams, P. A. 1982. Functional responses of optimal foragers. American Naturalist 120:382-390.

Altwegg, R., M. Eng, S. Caspersen, and B. R. Anholt. 2006. Functional response and prey defence level in an experimental predator-prey system. Evolutionary Ecology Research 8:115-128.

Anholt, B. R., D. Ludwig, and J. B. Rasmussen. 1987. Optimal pursuit times: how long should predators pursue their prey? Theoretical Population Biology 31:453-464.

Burnham, K. P., and D. R. Anderson. 2002. Model selection and multimodal inference: a practical information-theoretic approach. Springer, New York.

Dodson, S. I. 1974. Adaptive change in plankton morphology in response to size-selective predation: new hypothesis of cyclomorphosis. Limnology and Oceanography 19:721-729.

Gurney, W. S. C., and R. M. Nisbet. 1998. Ecological dynamics. Oxford University Press, Oxford.

Hammill, E., P. Kratina, and B. R. Anholt. 2009. Non-lethal presence of predators modifies morphology and movement rates in Euplotes. Hydrobiologia 621:183-189.

Hammill, E., P. Kratina, A. P. Beckerman, and B. R. Anholt. 2010. Precise time interactions between behavioural and morphological defences. Oikos 119:494-499.

Hassell, M. P., J. H. Lawton, and J. R. Beddington. 1977. Sigmoid functional responses by invertebrate predators and parasitoids. Journal of Animal Ecology 46:249-262.

Holling, C. S. 1959. Some characteristics of simple types of predation and parasitism. Canadian Entomologist 91:385-389.

Jeschke, J. M., and R. Tollrian. 2000. Density-dependent effects of prey defences. Oecologia (Berlin) 123:391-396.

Jeschke, J. M., M. Kopp, and R. Tollrian. 2002. Predator functional responses: discriminating between handling and digesting prey. Ecological Monographs 72:95-112.

- 2004. Consumer-food systems: why Type I functional responses are exclusive to filter feeders. Biological Reviews 79:337349.

Kratina, P., M. Vos, and B. R. Anholt. 2007. Species diversity modulates predation. Ecology 88:1917-1923.

Kusch, J. 1993. Behavioral and morphological changes in ciliates induced by the predator Amoeba proteus. Oecologia (Berlin) 96: 354-359.

Leary, D. J., and O. Petchey. 2009. Testing a biological mechanism of the insurance hypothesis in experimental aquatic communities. Journal of Animal Ecology 79:1143-1151.
Lehman, C. L., and D. Tilman. 2000. Biodiversity, stability, and productivity in competitive communities. American Naturalist 156: 534-552.

Lipcius, R. N., and A. H. Hines. 1986. Variable functional responses of a marine predator in dissimilar homogeneous microhabitats. Ecology 67:1361-1371.

Lively, C. M. 1986. Predator-induced shell dimorphism in the acorn barnacle Chthamalus anisopoma. Evolution 40:232-242.

Lotka, A. J. 1925. Elements of physical biology. Williams \& Wilkins, Baltimore.

R Development Core Team. 2009. R: a language and environment for statistical computing. R Foundation for Statistical Computing, Vienna.

Rasband, W. S. 2006. ImageJ. Version 1.36. U.S. National Institutes of Health, Bethesda, MD.

Real, L. A. 1977. The kinetics of functional response. American Naturalist 111:289-300.

Relyea, R. A. 2001. Morphological and behavioral plasticity of larval anurans in response to different predators. Ecology 82:523-540.

Sarnelle, O., and A. E. Wilson. 2008. Type III functional response in Daphnia. Ecology 89:1723-1732.

Schaffner, A. K., and B. R. Anholt. 1998. Influence of predator presence and prey density on behavior and growth of damselfly larvae (Ischnura elegans) (Odonata: Zygoptera). Journal of Insect Behavior 11:793-809.

Sih, A. 1980. Optimal foraging: partial consumption of prey. American Naturalist 116:281-290.

Sinclair, A. R. E., R. P. Pech, C. R. Dickman, D. Hik, P. Mahon, and A. E. Newsome. 1998. Predicting effects of predation on conservation of endangered prey. Conservation Biology 12:564-575.

Skelly, D. K. 1994. Activity level and the susceptibility of anuran larvae to predation. Animal Behaviour 47:465-468.

Solomon, M. E. 1949. The natural control of animal populations. Journal of Animal Ecology 18:1-35.

Sugibayashi, R., and T. Harumoto. 2000. Defensive function of trichocysts in Paramecium tetraurlia against heterotrich ciliate Climacostomum virens. European Journal of Protistology 36:415-422.

Tollrian, R., and C. D. Harvell. 1999. The ecology and evolution of inducible defenses. Princeton University Press, Princeton, NJ.

Van Buskirk, J., and S. A. McCollum. 2000. Functional mechanisms of an inducible defence in tadpoles: morphology and behaviour influence mortality risk from predation. Journal of Evolutionary Biology 13:336-347.

Vos, M., B. W. Kooi, D. L. DeAngelis, and W. M. Mooij. 2004. Inducible defences and the paradox of enrichment. Oikos 105: 471-480.

Wagenmakers, E.-J., and S. Farrell. 2004. AIC model selection using Akaike weights. Psychonomic Bulletin and Review 11:192-196.

Werner, E. E., and B. R. Anholt. 1993. Ecological consequences of the trade-off between growth and mortality rates mediated by foraging activity. American Naturalist 142:242-272.

Wiackowski, K., and A. Staronska. 1999. The effect of predator and prey density on the induced defence of a ciliate. Functional Ecology 13:59-65. 